\title{
Towards High-power Single-cycle THz Laser for Initiating High-field-sensitive Phenomena
}

\author{
Clemens Rucherta, Fernando Ardana ${ }^{\text {ab }}$, Alexandre Trisorio ${ }^{a}$, Carlo Vicario ${ }^{a}$, and \\ Christoph P. Hauri ${ }^{\star a b}$
}

\begin{abstract}
Powerful THz radiation confined in one field period or less is an adequate tool for triggering nonlinear actions. We show results towards the realization of a tunable high-power $\mathrm{THz}$ source based on a laser-driven frequency conversion scheme in plasma and nonlinear crystals. A powerful $\mathrm{THz}$ source in combination with the future X-ray Free Electron Laser facility in Switzerland (SwissFEL) holds promise for exciting experiments in a variety of different research areas.
\end{abstract}

Keywords: SwissFEL $\cdot$ THz radiation $\cdot$ THz source

\section{Introduction}

$\mathrm{THz}$ radiation located between the optical and the microwave frequency region $(0.1-10 \mathrm{THz})$ is well suited to study fundamental physical phenomena and to drive potential applications in life science (e.g. investigation of collective modes in proteins), homeland security (e.g. detection of explosives) and medicine (e.g. subcutaneous imaging of skin cancer). In fundamental science coherent $\mathrm{THz}$ radiation is the appropriate tool to investigate, for example, electrons in highly-excited atomic Rydberg states, electron dynamics in semiconductors and in superconductors having the energy gaps at $\mathrm{THz}$ frequencies. Depending on the application the appropriate $\mathrm{THz}$ source varies from continuous-wave/ narrowband low peak power $\mathrm{THz}$ for highresolution spectroscopy experiments to ultra-broadband high-peak power half-cycle pulses for initiating high field phenomena.

The interest of our research group is the development of a compact laser-driven powerful half/single-cycle $\mathrm{THz}$ source synchronized to a femtosecond X-ray and

${ }^{*}$ Correspondence: Prof. C. P. Hauriab Tel.: +41563104197

E-mail: christoph.hauri@psi.ch

apaul Scherrer Institute

$\mathrm{CH}-5232$ Villigen

${ }^{b}$ Ecole Polytechnique de Lausanne

$\mathrm{CH}-1015$ Lausanne
IR laser. The source should deliver highest electro-magnetic fields, should be tunable in central frequency $(0.3-15 \mathrm{THz})$ and provide the appropriate field polarity on the sample. The intended electric and magnetic field strengths exceeding MV/ $\mathrm{cm}$ and Tesla, respectively are suited for initiating high-field phenomena on surfaces $\left(e . g\right.$. catalytic reactions $\left.{ }^{[1]}\right)$, to study ultrafast magnetization dynamics and to realize a novel type of $\mathrm{THz}$-based streak camera $^{[2]}$ for characterizing sub-50 femtosecond X-ray pulses at SwissFEL, ${ }^{[3]}$ the Free Electron Laser facility at the Paul Scherrer Institute (Villigen, Switzerland). SwissFEL is planned to go into operation in 2017.

We started our laser-based THz activities at the Paul Scherrer Institute in late 2010. We are currently commissioning different $\mathrm{THz}$ generation schemes and a detection setup consisting of a bolometer, a Golay cell and an electro-optical sampling scheme for electric field reconstruction.

The goal of producing $\mathrm{THz}$ transients beyond the state of the art initiated the investigation of two different approaches based on nonlinear interaction in a plasma and on nonlinear organic crystals, respectively.

\section{Plasma-based THz Source}

The formation of $\mathrm{THz}$ radiation in a plasma can be explained in the frame of four-wave rectification according to $\left(2 \omega_{\mathrm{L}}\right.$ $\left.+\omega_{\mathrm{THz}}\right)-\omega_{\mathrm{L}}-\omega_{\mathrm{L}}=\omega_{\mathrm{THz}}$, with $\omega_{\mathrm{L}}, 2 \omega_{\mathrm{L}}$ and $\omega_{\mathrm{THz}}$ the fundamental, its second harmonic and the resulting $\mathrm{THz}$ frequency, respectively. This third-order nonlinear interaction is initiated by focusing an intense femtosecond laser pulse and its second

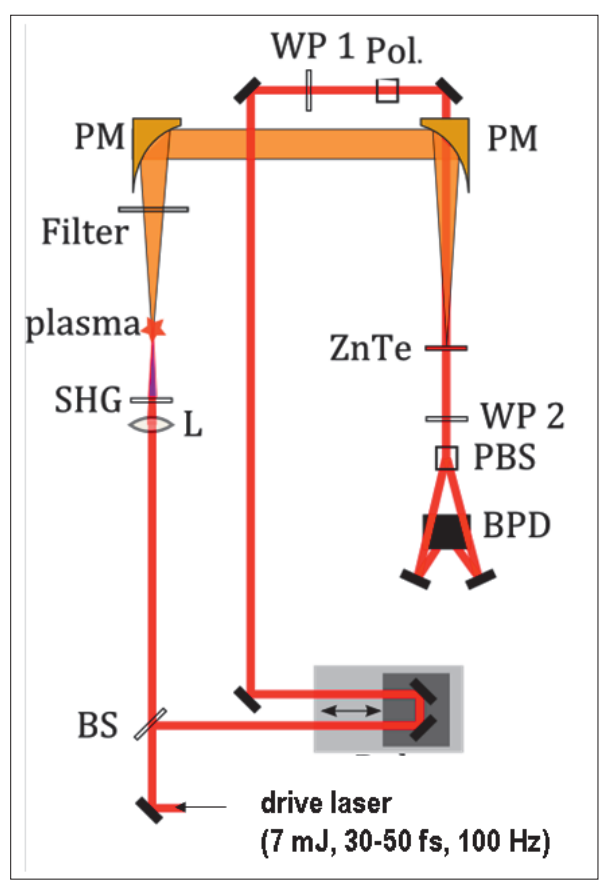

Fig. 1. The PSI four wave mixing $\mathrm{THz}$ source. Incident pump pulse with subsequent secondharmonic and probe pulse for optical sampling are delivered by a Ti:sapphire laser. Other components: half-wave plate (HWP), off-axis parabola (PM), optical sampling detection crystal (ZnTe), quarter wave plate (QWP) and balanced amplified photodetector (BPM).

harmonic in a gas. This nonlinear interaction has recently been demonstrated to give rise to strong $\mathrm{THz}$ emission. A schematic of our setup is shown in Fig. 1. As laser system we use a powerful Ti:sapphirebased amplifier system delivering up to $20 \mathrm{~mJ}, 20 \mathrm{fs}$ pulses at a repetition rate of $100 \mathrm{~Hz}$. The pulses are focused in air or argon by a lens ( $f=100 \mathrm{~mm}$ ) and frequency-doubled in a beta-barium borate (BBO, 


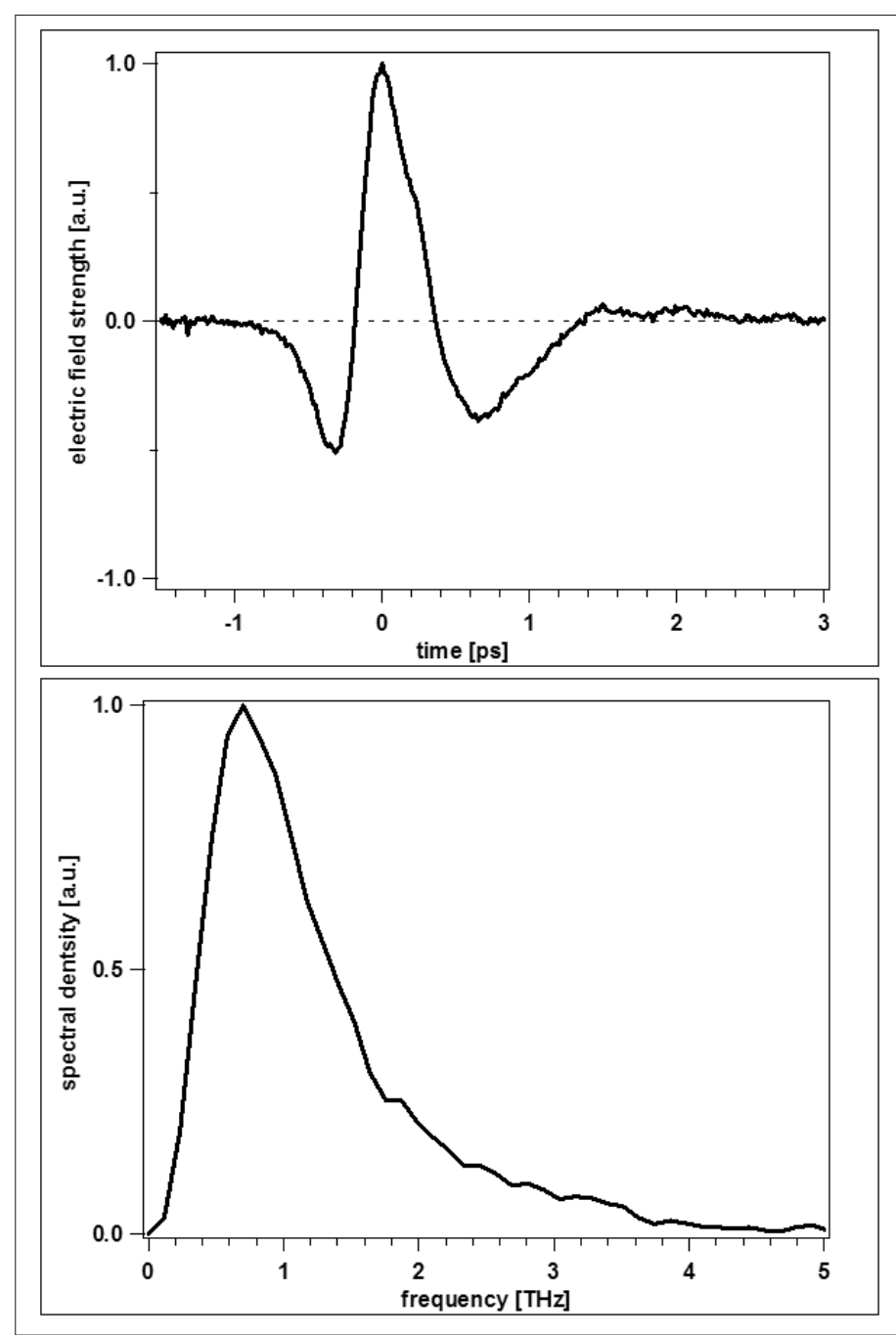

Fig. 2. An electrooptic sampling trace of the $\mathrm{THz}$ signal from the PSI optical rectification setup based on plasma interaction.
Fig. 3. Control of field polarization by introducing a delay between fundamental and second harmonic.

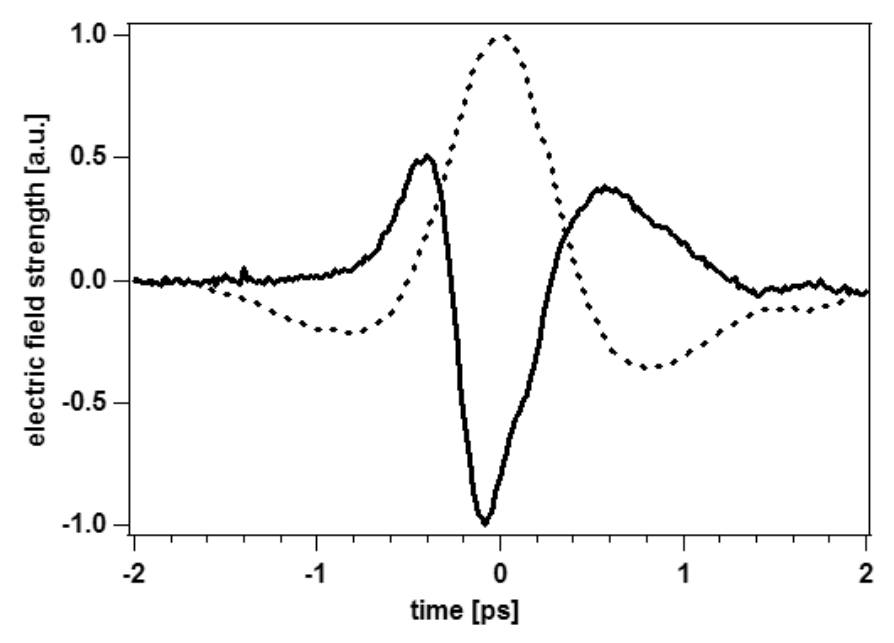

$0.1 \mathrm{~mm}$ ) nonlinear crystal. Typically $7 \mathrm{~mJ}$ pulse energy is used to drive the plasmabased THz scheme. Up to present we have achieved absolute field strengths of up to $430 \mathrm{kV} / \mathrm{cm}$ at a central frequency of 0.7 THz. Shown in Fig. 2 are the reconstructed spectrum and the electric field with only 1.5 cycles. These results are similar to recent published data for this type of $\mathrm{THz}$ source. ${ }^{[4]}$

Depending on the application it is important to provide the appropriate polarity and center frequency on the sample. The control of polarity (Fig. 3) can be achieved in our setup by changing the time delay between the fundamental and the second harmonic by a phase plate, for example. The polarity reverses for a delay corresponding to a half cycle of the second harmonic wave $(\approx 0.7 \mathrm{fs}$ for $400 \mathrm{~nm})$ with respect to the fundamental wave. A promising approach for controlling the $\mathrm{THz}$ central frequency could be the variation of the gas pressure. Similar to ref. [5] we are planning to in- vestigate a gas cell filled with rare gas at various pressures to reach a tunable $\mathrm{THz}$ field across $0.3-2 \mathrm{THz}$.

Unfortunately the plasma-based approach has limited potential for reaching higher field strength ( $>0.4 \mathrm{MV} / \mathrm{cm})$. Strong defocusing provoked by the free electrons in the plasma prevents the nonlinear mixing process from working at higher intensities in the interaction zone. We observed a clear saturation in $\mathrm{THz}$ yield for laser energies exceeding $7 \mathrm{~mJ}$. Up-scaling the electric field strengths toward $\mathrm{MV} / \mathrm{cm}$ seems challenging with the presented scheme.

\section{THz Generation in Nonlinear Crystals}

Typical field strengths of $\mathrm{THz}$ pulses generated by plasma-based sources reach $0.4 \mathrm{MV} / \mathrm{cm}$ but are usually limited to central frequencies below or around $1 \mathrm{THz}$. To overcome those limits we are exploring an alternative approach based on organic crystalline salts such as DAST (4-N,Ndimethylamino-4'-N'-methyl stilbazolium tosylate). ${ }^{[6]}$ These crystals exhibit electrooptical and nonlinear optical coefficients that are among the highest of all known materials ${ }^{[7]}$ and should allow efficient $\mathrm{THz}$ generation by optical rectification. Pumped with an infrared wavelength of typically $1.3 \mu \mathrm{m} \mathrm{THz}$ radiation is generated in DAST by optical rectification. Presently up to $4 \mathrm{THz}$ has been reported at rather low electric field strength (up to 80 $\mathrm{kV} / \mathrm{cm})^{[6]}$ mainly due to limited power in the infrared pump (several tens of $\mu \mathrm{J}$ ). Our in-house multi-stage optical parametric amplifier provides up to several $\mathrm{mJ}$ at 1.3 $\mu \mathrm{m}$ wavelength. This laser is presently being used to explore $\mathrm{THz}$ radiation in DAST by optical rectification. A typical electrooptical trace with the corresponding spectrum is shown in Fig. 4. Frequencies up to $5 \mathrm{THz}$ are generated and the measured field strength reaches $600 \mathrm{kV} / \mathrm{cm}$ and more. The field has been reconstructed by electrooptical sampling in GaP with an $800 \mathrm{~nm}$ laser pulse. Our first results indicate that optical parametric rectification in DAST is a promising candidate for high electric field. Up-scaling of the present scheme to higher electric fields exceeding $1 \mathrm{MV} / \mathrm{cm}$ seems feasible by scaling the crystal size and pump energy.

\section{THz Transportation}

Pulses with a spectral content $>1 \mathrm{THz}$ suffer from a significant drop in field strength when propagating in ambient air. Numerous absorption lines are present originating from ambient water vapour in 


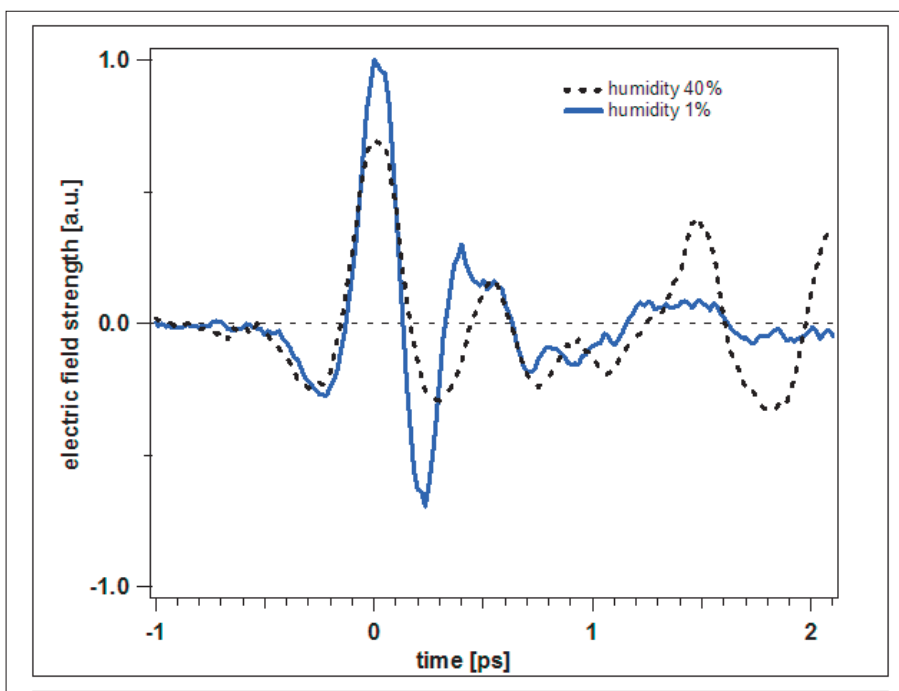

Fig. 4. Electric field and corresponding spectrum generated by optical rectification in DAST after propagation in dry air (solid line) and in ambient air (dashed line).

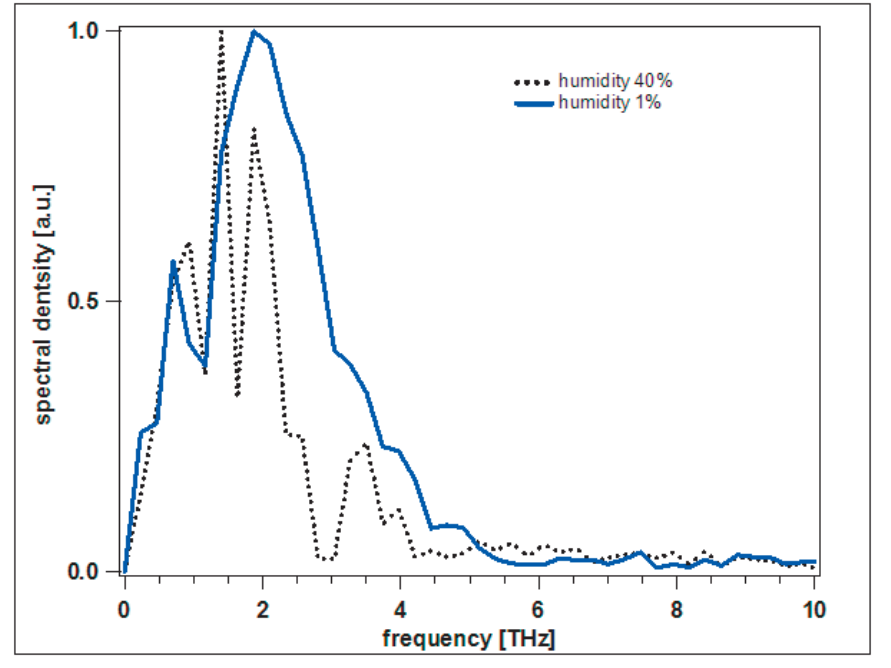

our laboratory (40\% humidity, $20^{\circ} \mathrm{C}$ ). ${ }^{[8]}$ In our setup the water absorptions lead to temporal pulse distortion and a significant drop of the electric field by $30 \%$ for a propagation distance of $0.5 \mathrm{~m}$ (Fig 4). Transporting high-field $\mathrm{THz}$ pulses from the source to the target requires therefore careful design of transport beam lines either in vacuum or flooded with dry air or nitrogen to avoid re-absorption of $\mathrm{THz}$ radiation.

In conclusion we have presented our activities towards developing a laser-driven high-power single-cycle $\mathrm{THz}$ source based on optical rectification in a plasma and organic crystals. The presented schemes should allow the generation of electric and magnetic field strengths beyond state of the art.

\section{Acknowledgements}

The authors would like to thank B. Patterson, J. Sa, A. Ichsanow, I. Czekay and J. van Bokhoven for ongoing fruitful discussions and the PSI-SwissFEL team for their support. We acknowledge support from the NCCR MUST program of the Swiss National Science Foundation.
[1] B. D. Patterson, J. Sa, A. Ichasanow, C. Hauri, C. Ruchert, I. Czekay, R. Gehrig, H. C. Sigg, J. A. van Bokhoven, B. Pedrini, R. Abela, Chimia 2011, 65, 323.

[2] U. Fruehling, M. Wieland, M. Gensch, T. Gebert, B. Schuette, M. Krikunova, R. Kalms, F. Budzyn, O. Grimm, J. Rossbach, E. Ploenjes, M. Drescher, Nature Photonics 2009, 3, 523 .

[3] B. D. Patterson, R. Abela, H. H. Braun, U. Flechsig, R. Ganter, Y. Kim, E. Kirk, A. Oppelt, M. Pedrozzi, S. Reiche, L. Rivkin, T. Schmidt, B. Schmitt, V. N. Strocov, S. Tsujino, A. F. Wrulich, New J. Phys. 2010, 12, 035012.

[4] T. Bartels, P. Gall, K. Reimann, M. Woerner, T. Elsaesser, Opt. Lett. 2005, 30, 2805.

[5] N. Karpowicz, X. Lu, Z. C. Zhang, J. Mod. Opt. 2009, 56, 1137.

[6] A. Schneider, M. Neis, M. Stillhart, B. Ruiz, R. U. A. Khan, P. Guenter, JOSA B 2006, 23, 1822.

[7] F. Pan, G. Knoepfle, C. Bosshard, S. Follinier, R. Spreter, M. S. Wong, P. Guenter, Appl. Phys. Lett. 1996, 69, 13.

[8] http://spec.jpl.nasa.gov 